\title{
Numerical Simulation on the Anti-fouling Property of Helical Blade Rotors in Circular Tube
}

\author{
Lichen He, Weimin Yang, Changfeng Guan, Hua Yan \\ College of Mechanical and Electrical Engineering, Beijing University of Chemical Technology \\ Chaoyang District, North Third Ring Road 15, Beijing 100029, China \\ 2015400117@mail.buct.edu.cn
}

\begin{abstract}
Inferior heat transfer efficiency and severe fouling precipitation have been the troublesome problems for shell-and-tube heat exchangers. As a typical type of tube insert, the helical blade rotors (HBRs) can achieve heat transfer augmentation and fouling mitigation in heat exchanger. In this work, the numerical simulation regarding to the anti-fouling property of HBRs in circular tube was conducted. The numerical method was verified accurate in comparison with the results in literature prior to the simulation. The results showed that in the process of the crystallization of the fouling the deposition rate decreased and the removal rate increased, and eventually they tended to be stable and reached a dynamic balance; the deposition rate, the removal rate, the net rate and the thermal resistance in tube with HBRs was lower than that in smooth tube, which means that inserting HBRs into the circular tube can effectively prevent the deposition of crystallization fouling; moreover, the mass fraction of $\mathrm{CaCl}_{2}$ in tube with $\mathrm{HBRs}$ was lower than that in smooth tube, and in tube with HBRs it was not just lower near the tube wall but also near the HBRs, which illustrate that the HBRs can enhance the mass transfer of $\mathrm{Ca}^{2+}$ in circular tube, and the mass transfer of $\mathrm{Ca}^{2+}$ towards the HBRs is enhanced, while the mass transfer towards the tube wall is weakened.
\end{abstract}

Keywords: Heat and Mass Transfer, Crystallization Fouling, Helical Blade Rotors (HBRs), Computational Fluid Dynamics (CFD).

\section{Introduction}

Shell-and-tube heat exchangers, as one kind of high energy consuming equipment, are extensively applied in fields of chemical production, petroleum refinery, power generation and metallurgy, and so on. However, the inferior heat transfer efficiency and the severe fouling precipitation have been the intractable problems disturbing enterprises and even industries and have caused large amounts of energy loss. Therefore, it is no time to delay to improve this situation by taking effective measures.

The heat transfer enhancement technology is just an effective approach to resolve the problem. Heat transfer enhancement techniques can be classified either as passive, which require no direct application of external power, or as active, which require external power[1]. In comparison with the active techniques, the passive ones are applied more widely in engineering. A variety of passive techniques have been developed, among which the tube inserts are the most popular ones, this is because they can be installed and taken down with ease in existing tube bundles during the routine maintenance without the need for replacing them. The tube inserts consist of several common types: coiled wire[2], static mixer[3], vortex generator[4], and twisted tape[5].

In the aspect of fouling mitigation with tube inserts, a few of meaningful research work has been carried out. Han et al. [6] numerically studied the deposition process of $\mathrm{CaSO}_{4}$ fouling in the rectangular channel with half-cylinder vortex generators and investigated the fouling characteristics under different operation conditions and structures such as the concentration of $\mathrm{CaSO}_{4}$, the wall temperature, the inlet velocity, the length, the radius and the spacing of the half-cylinder vortex generators. Hasan et al. [7] carried out an experiment to study the effect of surface enhancement on the crystallization fouling in a double pipe heat exchanger by installing a coiled wire insert on the inside or outside surface of the inner tube. Pahlavanzadeh et al. [8] investigated the effect of two tube inserts (wire coil and wire mesh) on the heat transfer enhancement, pressure drop and mineral salts fouling mitigation in tube of a heat exchanger. The results revealed that wire coil insert with vibration mitigated mineral salts fouling (scaling) about 34\%, and wire mesh had no effect on scaling. Yang et al. [9] investigated a cleaner-augmenter (a spiral wire) on the internal surface of a tube. The results 
showed that the cleaner-augmenter enhanced heat transfer in single phase flow and removed fouling from the heat exchange surface.

As a novel kind of tube insert, helical blade rotors (HBRs) was proposed by Yang et al. [10]. The installation of HBRs in circular tube is shown in Fig.1. When water flows through the tube, it could impact the HBRs rotate around the support shaft to achieve heat transfer augmentation and scale prevention.

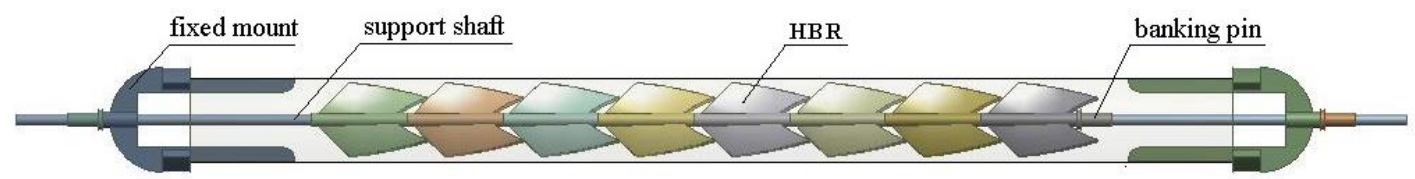

Fig.1: Installation of HBRs in circular tube.

With regard to the thermo-hydraulic characteristics of the HBRs, much research has been done[11-13], while for the fouling mitigation performance, attention has been paid insufficiently. The formation of crystallization fouling is a complex physicochemical process that contains heat and mass transfer, and the numerical simulation (CFD) has great advantage in studying such process. Therefore, in this work we adopt the numerical method to investigate the mass rate, thermal resistance and concentration distribution in circular tube inserted with HBRs to reveal the anti-fouling property of the HBRs.

\section{Numerical Simulation}

\subsection{Geometric Model}

The geometrical models ofthe circular tube and the HBR are built with SOLIDWORKS, and theirgeometrical parametersare shown in Fig.2. The circular tube has a length of $1500 \mathrm{~mm}$, and a diameter of $\varnothing 24 \mathrm{~mm}$. The length of the HBR is $27.5 \mathrm{~mm}$, the diameter of the central axis is $4 \mathrm{~mm}$, and the rotational diameter of the blade is $20 \mathrm{~mm}$. Fifty HBRs in series are inserted into the circular tube. The dimensions of the smooth tube for comparison are the same with that of the HBRs-equippedcircular tube.

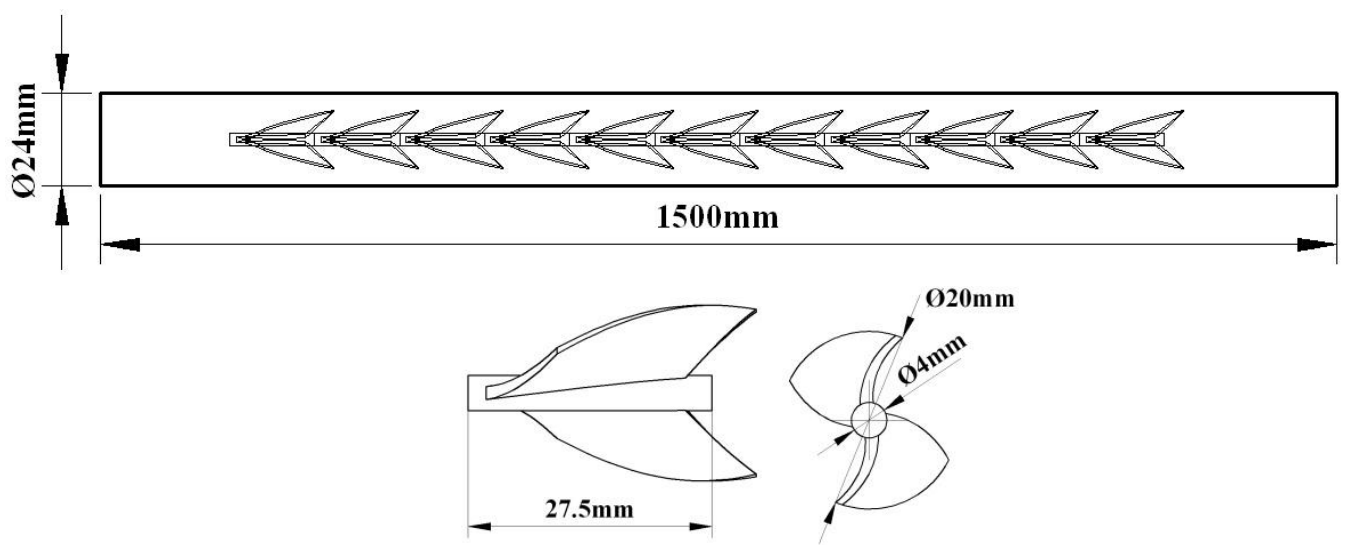

Fig.2: Geometrical parameters of the circular tube and the HBR.

\subsection{Governing Equation}

The governing equations for continuity, momentum, energy and species can generally be expressed as follows:

$$
\frac{\partial(\rho \phi)}{\partial t}+\operatorname{div}(\rho U \phi)=\operatorname{div}\left(\Gamma_{\phi} \operatorname{grad} \phi\right)+S_{\phi}
$$


Where, $\phi$ is the generalized variable, and it represents the variables of $u, v, w, T, c ; \Gamma_{\phi}$ is the generalized diffusion coefficient, and $S_{\phi}$ is the generalized source term.

\subsection{Solution Methods and Boundary Conditions}

The geometric and mathematical models are numerically solved in the commercial software ANSYS FLUENT 14.0. The standard k- $\varepsilon$ turbulence model and standard wall functions are selected to calculate the flow field and temperature field in the circular tube, and the species transport model and wall surface reaction are used for solving the concentration field. The pressure-velocity coupling scheme is SIMPLE. The spatial discretization method for pressure is standard, and for momentum, turbulent kinetic energy $(\mathrm{k})$, turbulent dissipation rate $(\varepsilon)$, species and energy, it is second order upwind. The convergence criterion of residual for continuity is $1 \times 10^{-4}$, and residuals for other variables are $1 \times 10^{-6}$.

At the inlet, it is the velocity inlet boundary condition: the velocity magnitude is $0.3686 \mathrm{~m} / \mathrm{s}$ (equivalent to volume flow rate $0.6 \mathrm{~m}^{3} / \mathrm{h}$ ), the temperature is $300 \mathrm{~K}$, and the concentration of the $\mathrm{CaCO}_{3}$ solution is $800 \mathrm{mg} / \mathrm{L}$; at the outlet, it is the outflow boundary condition; at the tube wall, it is the no slip stationary wall, and the wall temperature is $320 \mathrm{~K}$; at the wall of rotors, it is the moving wall, the rotational speed of the rotors is acquired by experiment, and it is adiabatic at the solid/fluid interface.

The crystallization model of $\mathrm{CaCO}_{3}$ employed in this work is same with that in literature[14]. The time step $\Delta t$ is selected as 3600s, which is considered to be appropriate for deposition process of the fouling. Within the first time step $\mathrm{t}=0-3600 \mathrm{~s}$, the mass of the deposited fouling is $m=\dot{m}_{d} \Delta t$, the mass removal rate $\dot{m}_{r}=0$, afterwards the mass of the deposited fouling is calculated by equations (2) and (3):

$$
\begin{gathered}
m_{t+\Delta t}=m_{t}+\dot{m} \Delta t \\
\dot{m}=\dot{m}_{d}-\dot{m}_{r}
\end{gathered}
$$

The mass removal rate $\dot{m}_{r}$ is calculated from equation (4):

$$
\dot{m}_{r}=\frac{K}{P} \rho_{f}(1+\beta \Delta T) d_{p}\left(\rho^{2} \mu g\right)^{1 / 3} \delta_{f} u^{2}
$$

The thermal resistance of the fouling can be modeled as:

$$
R_{f}=m /\left(\rho_{f} \lambda_{f}\right)
$$

Where, $\lambda_{f}$ is the thermal conductivity of the fouling, and $\rho_{f}$ is the density of the fouling.

\subsection{Grid independence}

The geometrical models are meshed with tetrahedron elements using ANSYS MESHING. To ensure the accuracy of the simulation, a careful check of the grid independence is carried out. A finer boundary layer grid is applied at the tube wall. The numerical result of the circular tube inserted with HBRs with different grid number is shown in Fig.3. Considering both solution precision and computational cost, the grid with 3215222 elements is adopted. 


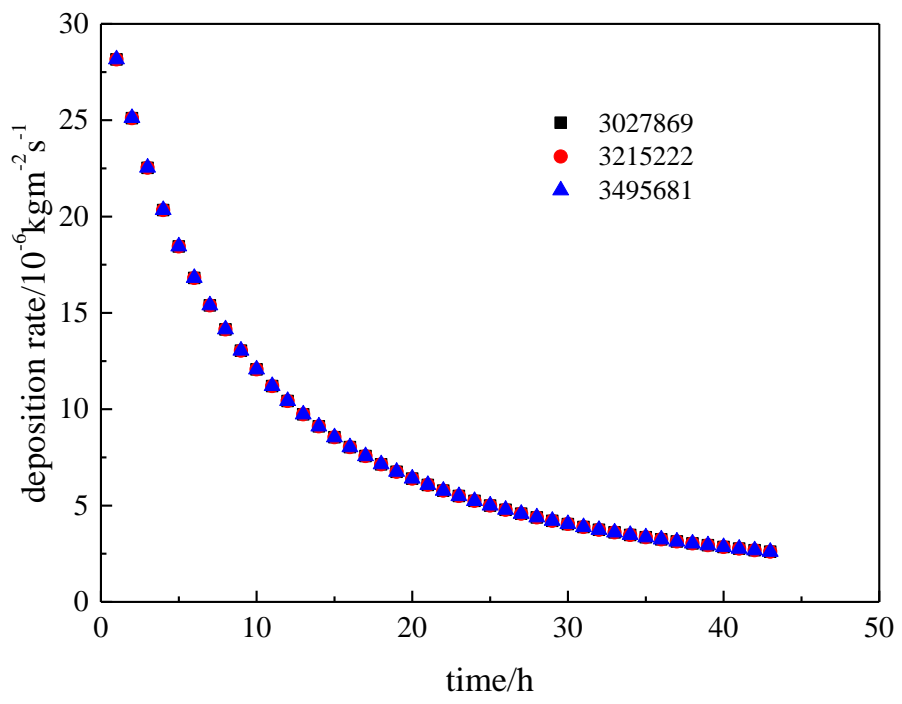

Fig.3: Check of grid independence.

\section{Results and Discussion}

\subsection{Verification of Numerical Method}

The verification of the numerical results in smooth tube is shown in Fig.4, among which (a) is the results in this work and (b) is the results in literature[14]. The results are acquired in the circular tube that has a length of $2200 \mathrm{~mm}$ and a diameter of $22 \mathrm{~mm}$, and the operating conditions of the two cases are the same. It can be seen that the results in this work match well with that in literature, which demonstrates that the numerical method used in this work is reliable.
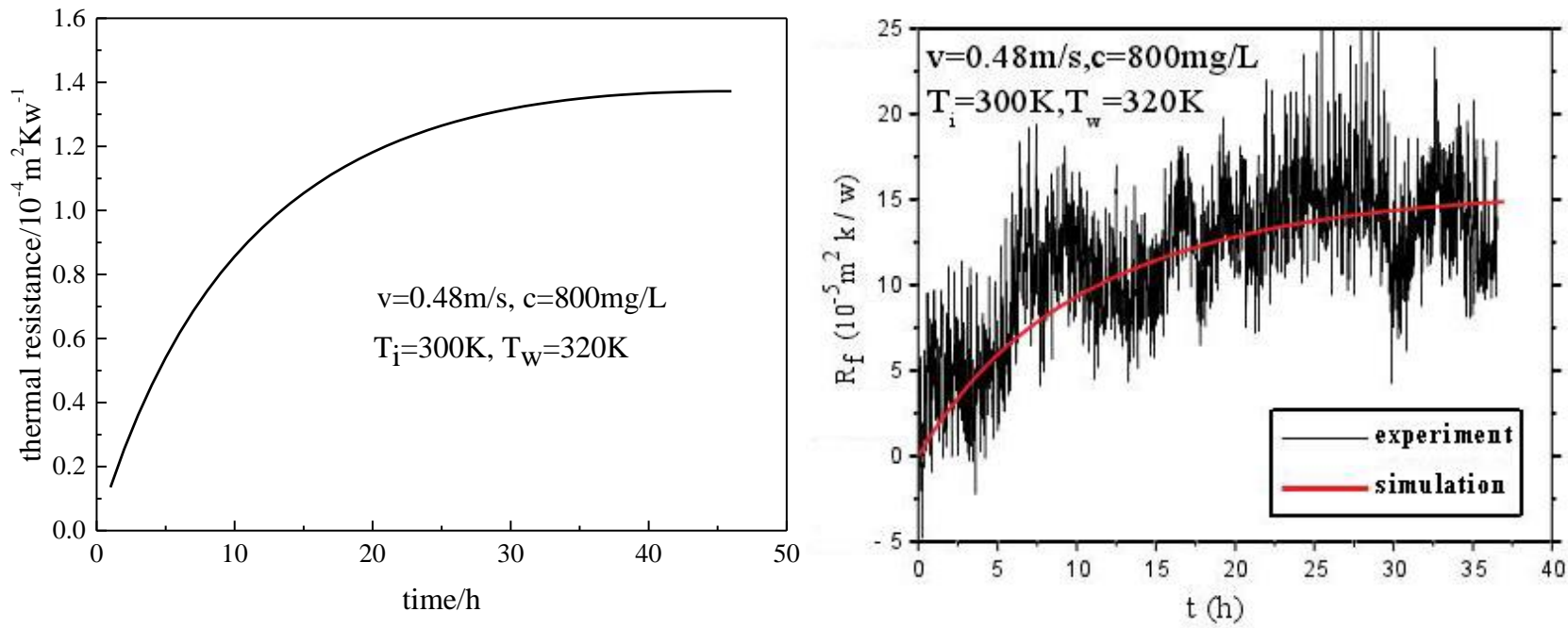

(a) Results in this work(b) Results in literature

Fig.4: Verification of the numerical results. 


\subsection{Mass Rate and Thermal Resistance}

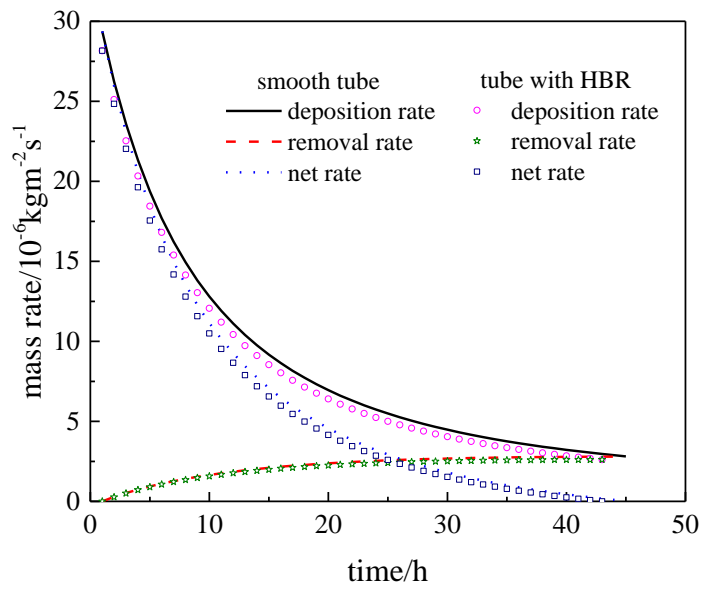

Fig.5: Mass rate of crystallization fouling.

The mass rate of crystallization fouling in tube with HBRs and smooth tube is shown in Fig.5. It can be seen that at the beginning the deposition rate is high and the removal rate is low, while with the increase of time the deposition rate decreases and the removal rate increases. The similarity which the deposition rate and the removal rate exhibit is that they both gradually slow down and eventually tend to be stable with the increase of time, which means that the deposition rate and the removal rate ultimately reach a dynamic balance. As is described in equation (3), the net rate is equal to the deposition rate subtracted by the removal rate, so we can see that the net rate gradually decreases and approximates zero when the deposition rate and the removal rate become stable. The results coincide with the whole physical process of the crystallization of the fouling.

We can also see that the deposition rate, the removal rate and the net rate in tube with HBRs are all lower than that in smooth tube. The reason why the removal rate is lower is that the removal rate is proportional to the total mass of the fouling of the previous time step, and the total mass of the fouling of the previous time step is primarily determined by the deposition rate. In fact, the removal rate in tube with HBRs is supposed to be higher than that in smooth tube, this is because when the HBRs are inserted into the circular tube, the velocity of the fluid in annular space between the HBRs and the tube wall would be increased, which undoubtedly results in a higher shear force and larger flushing action on the fouling deposited on the tube wall, meanwhile the ceaseless rotation of the HBRs can to some extent scrape the fouling. But the destruction on the fouling, which is brought about by the HBRs, is hard to be characterized quantificationally. So we assume that the removal rate is still depicted in equation (4), and we only focus on the deposition rate. Anyway, the net rate in tube with HBRs is lower than that in smooth tube.

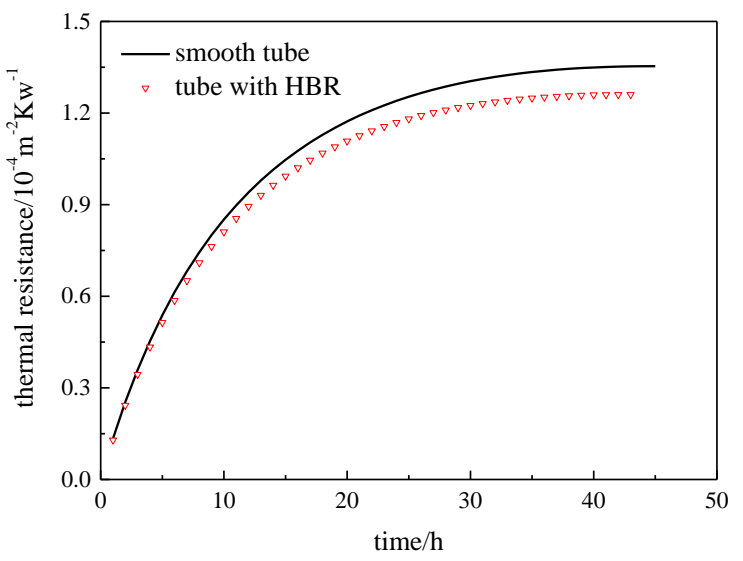

Fig.6: Thermal resistance of crystallization fouling 
Fig.6 shows the thermal resistance of crystallization fouling in tube with HBRs and smooth tube. As we can see, with the increase of time the thermal resistance of the fouling gradually increases until it reaches the maximum, the reason for this result is that the thermal resistance is directly derived from the mass of the fouling, and the mass of the fouling accumulates constantly with the increase of time, and when the net rate is close to zero, the thermal resistance maintains stable. We can also see that the maximum value of the thermal resistance is lower in tube with HBRs than in smooth tube when it is stable, which means that inserting HBRs into the circular tube can effectively prevent the deposition of crystallization fouling.

\subsection{Temperature and Concentration Distribution}

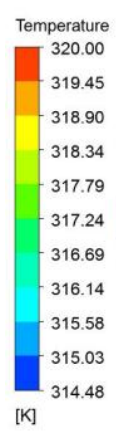

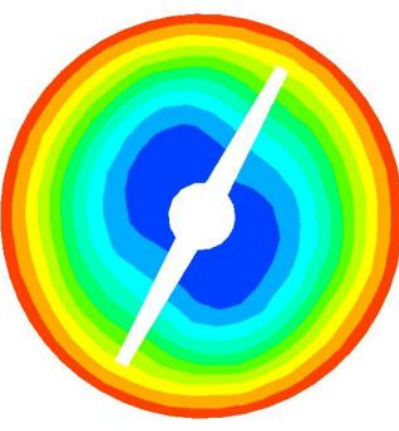

(a) Tube with HBRs

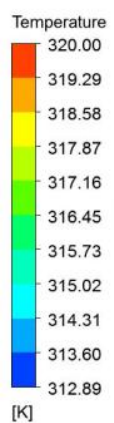

(b) Smooth tube

Fig.7: Temperature distribution.

The temperature distributions in tube with HBRs and smooth tube are shown in Fig.7. We can see that the cold fluid in tube with HBRs is distributed more even compared with that in smooth tube, and the minimum temperature is higher in tube with HBRs than in smooth tube, which indicates that the heat exchange between the cold fluid in the tube and the heated tube wall is more intense, this is because the rotation of the HBRs can promote the mixing of the fluid in tube, thus more cold fluid could be directly involved in the heat transfer between the fluid and the tube wall.

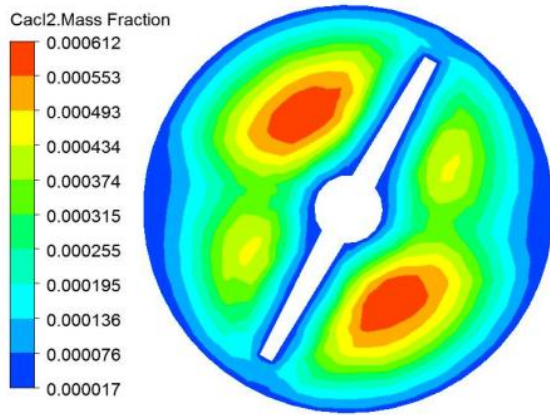

(a) Tube with HBRs

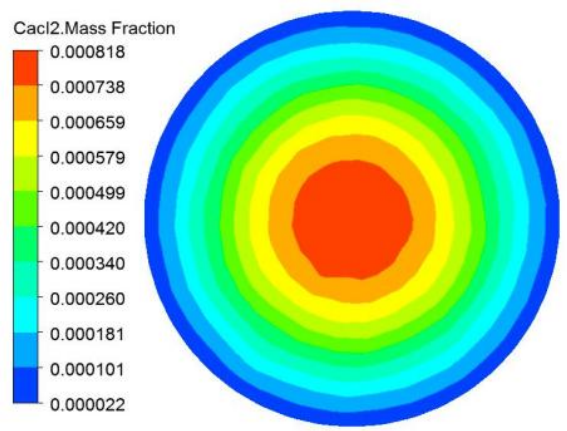

(b) Smooth tube

Fig.8: Concentration distribution of $\mathrm{CaCl}_{2}$.

The concentration distributions of $\mathrm{CaCl}_{2}$ in tube with HBRs and smooth tube are shown in Fig.8. The concentration distribution illustrates the mass transfer state of $\mathrm{CaCl}_{2}$. It can be seen that the maximum value of the mass fraction of $\mathrm{CaCl}_{2}$ in tube with HBRs is distinctly lower than that in smooth tube, which demonstrates that much more $\mathrm{Ca}^{2+}$ in mainstream have been deposited in the form of $\mathrm{CaCO}_{3}$ in tube with HBRs than in smooth tube, that is the HBRs can enhance the mass transfer of $\mathrm{Ca}^{2+}$ in circular tube. We can also see that the concentration of $\mathrm{Ca}^{2+}$ in tube with HBRs is not just lower near the tube wall, but also near the HBRs, compared with that in smooth tube. This result means that the $\mathrm{CaCO}_{3}$ crystallizes at both the tube wall and the wall of HBRs, and the $\mathrm{Ca}^{2+}$ in mainstream would transfer towards the tube wall as well as the HBRs. 
However, the deposition rate of the crystallization fouling $\mathrm{CaCO}_{3}$ is lower in tube with HBRs than in smooth tube, which is acquired in Fig.5,therefore we can conclude that the mass transfer of $\mathrm{Ca}^{2+}$ towards the HBRs is enhanced by the HBRs, while the mass transfer towards the tube wall is weakened.

\section{Conclusions}

In this work, the numerical simulation regarding to the anti-fouling property of HBRs in circular tube was conducted and the temperature and concentration distributions in tube with HBRs and smooth tube were presented. The conclusions can be drawn as follows:

In the process of the crystallization of the fouling, the deposition rate decreased and the removal rate increased, and eventually they tended to be stable and reached a dynamic balance; the deposition rate, the removal rate, the net rate and the thermal resistance in tube with HBRs was lower than that in smooth tube, which means that inserting HBRs into the circular tube can effectively prevent the deposition of crystallization fouling; in addition, the mass fraction of $\mathrm{CaCl}_{2} \mathrm{in}$ tube with HBRs was lower than that in smooth tube, and in tube with HBRs it was not just lower near the tube wall but also near the HBRs, which illustrate that the HBRs can enhance the mass transfer of $\mathrm{Ca}^{2+}$ in circular tube, and the mass transfer of $\mathrm{Ca}^{2+}$ towards the HBRs is enhanced, while the mass transfer towards the tube wall is weakened.

\section{References}

[1] A.E. Bergles, "ExHFT for fourth generation heat transfer technology," Exp Therm Fluid Sci, vol. 26, pp. 335-344, 2002.

[2] K. Sharifi, M. Sabeti, M. Rafiei, A.H. Mohammadi, L. Shirazi, "Computational fluid dynamics (CFD) technique to study the effects of helical wire inserts on heat transfer and pressure drop in a double pipe heat exchanger,"Appl Therm Eng, vol.128,pp. 898-910, 2018.

[3] Y. Qi, Y. Kawaguchi, R.N. Christensen, J.L. Zakin, "Enhancing heat transfer ability of drag reducing surfactant solutions with static mixers and honeycombs,"Int J Heat Mass Tran, vol.46, no.26, pp. 5161-5173, 2003.

[4] S. Chamoli, R. Lu, P. Yu, "Thermal characteristic of a turbulent flow through a circular tube fitted with perforated vortex generator inserts,"Appl Therm Eng, vol.121,pp. 1117-1134, 2017.

[5] C. C. Zhang, D. B. Wang, K. Ren, Y. Han, Y. J. Zhu, X. Peng, J. Deng, X. Y. Zhang, "A comparative review of selfrotating and stationary twisted tape inserts in heat exchanger,"Renew Sust Energ Rev, vol.53,pp. 433-449, 2016.

[6] Z. M. Han, Z. M. Xu, J. T. Wang, "CaSO ${ }_{4}$ fouling characteristics on the rectangular channel with half-cylinder vortex generators,"Appl Therm Eng, vol.128, 1456-1463, 2018.

[7] B.O. Hasan, E.A. Jwair, R.A. Craig, "The effect of heat transfer enhancement on the crystallization fouling in a double pipe heat exchanger,"Exp Therm Fluid Sci, vol.86,pp. 272-280, 2017.

[8] H. Pahlavanzadeh, M.R. Jafari Nasr, S.H. Mozaffari, "Experimental study of thermo-hydraulic and fouling performance of enhanced heat exchangers,"Int Commun Heat Mass, vol.34, no. 7, pp. 907-916, 2007.

[9] S.R. Yang, J.M. Wang, G.D. Zai, "Investigation of a Heat Transfer Augmenter as a Fouling Cleaner and Its Optimum Geometry in the Tube Side of a Condenser,"Exp Therm Fluid Sci, vol.5,pp.795-802, 1992.

[10] W.M. Yang, Y.M. Ding, L.B. Geng, W. Huang, "Rotor-assembled automatic cleaning and heat transfer enhancement device,"CN200520127121.9, 2005.

[11] Z. Zhang, W. M. Yang, C. F. Guan, Y. M. Ding, H. Yan, "Numerical study on thermo-hydraulic characteristics of turbulent flow in a circular tube fitted with helical blade rotors,"Int J Heat Mass Tran, vol. 60, pp. 603-611, 2013.

[12] P. Jiang, W. M. Yang, H. Yan, C. F. Guan, "Performance assessment in a tube with rotor-assembled strands mixed by spiral slot rotor and low flow resistance rotor,"Appl Therm Eng, vol. 52, no. 1, pp. 69-76, 2013.

[13] Z. Zhang, H. Yan, W. M. Yang, Y. P. Zhou, Y. M. Ding, C. F. Guan, "Heat transfer enhancement in the tube fitted with Left-Right helical blade rotors,"Appl Therm Eng, vol. 55, no. 1-2, pp. 95-101, 2013.

[14] Z. M. Xu, Z. B. Zhang, H. M. Cheng, "Numerical simulation of $\mathrm{CaCO}_{3}$ fouling process in a tube,"J EngTherm (in chinese), vol. 30, no. 12,pp. 2099-2101, 2009. 